Article

\title{
Oscillation Criteria for Third Order Neutral Generalized Difference Equations with Distributed Delay
}

\author{
P. Venkata Mohan Reddy ${ }^{1}\left(\right.$, M. Maria Susai Manuel ${ }^{1}\left[\right.$ and Adem Kılıçman ${ }^{2, *(\mathbb{C})}$ \\ 1 Department of Science and Humanities, R. M. D. Engineering College, Kavaraipettai 601206, Tamil Nadu, \\ India; vmr.maths@gmail.com (P.V.M.R.); mmariasusai@gmail.com (M.M.S.M.) \\ 2 Department of Mathematics and Institute for Mathematical Research, University Putra Malaysia, \\ Serdang 43400 UPM, Selangor, Malaysia \\ * Correspondence: akilic@upm.edu.my; Tel.: +603-89466813
}

Received: 19 November 2019; Accepted: 9 December 2019; Published: 11 December 2019

\begin{abstract}
This paper aims to investigate the criteria of behavior of a certain type of third order neutral generalized difference equations with distributed delay. With the technique of generalized Riccati transformation and Philos-type method, we obtain criteria to ensure convergence and oscillatory solutions and suitable examples are provided to illustrate the main results.
\end{abstract}

Keywords: generalized difference operator; oscillation; non-oscillation; converge to zero; distributed delay; Riccati transformation

\section{Introduction}

Difference equations and functional equations usually occur due to certain phenomena over time and play essential roles in the field of discrete dynamical systems [1]. Difference equations and their associated operators play a vital role as direct mathematical models of physical phenomena but also provide powerful tools in numerical methods. Given a differential equation with symmetries, we can construct a difference scheme, which appropriates it while preserving the symmetries. Difference equations play an important role in Lie theory. An important and significant observation is that difference equations either appear in themselves and they can be used in Lie theory to get classes of exact solutions, or they can be obtained by discretizing the continuum equation in such a way to preserve the symmetries. That is, we can create sets of discrete equations which provide numerical schemes approximating the continuum equations. For a detailed study on this aspect one can refer to $[2-5]$.

In this article, we consider the neutral generalized difference equation with distributed delay of the form

$$
\begin{aligned}
\Delta_{\ell}\left(p_{1}(\lambda) \Delta_{\ell}\right. & \left.\left(p_{2}(\lambda) \Delta_{\ell}\left(y(\lambda)+\sum_{s=a}^{b} g(\lambda, s) y(\lambda+s \ell-\tau \ell)\right)\right)\right) \\
& +\sum_{s=c}^{d} h(\lambda, s) f(y(\lambda+s \ell-\sigma \ell))=0,
\end{aligned}
$$

and analyze the behavior of its solutions. Here, $\Delta_{\ell}$ is the forward generalized difference operator defined by $\Delta_{\ell} y(\lambda)=y(\lambda+\ell)-y(\lambda), \mathbb{N}_{\ell}\left(\lambda_{0}\right)=\left\{\lambda_{0}, \lambda_{0}+\ell, \lambda_{0}+2 \ell, \cdots\right\}, \lambda_{0} \in[0, \infty), \ell \in(0, \infty)$ and $a, b, c, d \in \mathbb{N}\left(\lambda_{0}\right)$ are being assumed. 
$\left(c_{1}\right)\left\{p_{i}(\lambda)\right\}$ is a positive real sequence with $\sum_{\lambda=\lambda_{0}}^{\infty} \frac{1}{p_{i}(\lambda)}=\infty$, and $p_{i}(\lambda)>0$, for $i=1,2$;

$\left(c_{2}\right)\{g(\lambda, s)\}$ and $\{h(\lambda, s)\}$ are non-negative real sequences with $0 \leq \sum_{s=a}^{b} g(\lambda, s) \equiv g(\lambda) \leq g<1$;

(c) $f: \mathbb{R} \rightarrow \mathbb{R}$ is a continuous function such that $\frac{f(y)}{y} \geq L>0$, for $y \neq 0$;

$\left(c_{4}\right) m_{i}(\lambda)=\left[\frac{\lambda-\lambda_{i}-j-\ell}{\ell}\right], \bar{\lambda}=\lambda+j$ and $j=\lambda-\lambda_{i}-\left[\frac{\lambda-\lambda_{i}}{\ell}\right] \ell$.

Define a function $x(\lambda)$ by

$$
y(\lambda)+\sum_{s=a}^{b} g(\lambda, s) y(\lambda+s \ell-\tau \ell)=x(\lambda)
$$

We consider only those solutions $\{y(\lambda)\}$ of Equation (1) which satisfy $\sup \{|y(\lambda)|: \lambda \geq T\}>0$ for $\lambda \in \mathbb{N}_{\ell}\left(\lambda_{0}\right)$. A solution of Equation (1) is said to be non oscillatory if it is either eventually positive or negative and oscillatory otherwise. The generalized difference operator defined for any real sequence $\{y(\lambda)\}$ by $\Delta_{\ell} y(\lambda)=y(\lambda+\ell)-y(\lambda) \equiv z(\lambda)$, then its inverse is defined by

$$
y(\lambda)=y\left(\lambda_{0}+j\right)+\sum_{r=0}^{m_{0}(\lambda)} z\left(\lambda_{0}+j+r \ell\right) .
$$

Recently, many authors obtained certain behaviors of nonlinear difference equations and their applications. See for example monographs by Agarwal [6], Gyori and Ladas [7], and Elaydi [8]. The study of third order delay difference equations has also received much attention. The oscillatory and asymptotic behaviors of solutions of the third order difference equations were studied by Schmeidal [9]. Behaviors of oscillation of the third order nonlinear delay difference equation by Riccati transformation technique were obtained by several authors like Aktas et al. [10], Elabbasy et al. [11], Saker et al. [12], Selvaraj et al. [13-15], Thandapani et al. [16].

Here, we obtain conditions for the existence of convergent oscillatory solutions of Equation (1) with the help of the generalized Riccati transformation. In fact, by choosing an appropriate function, we shall present several oscillation criteria easily. The technique adopted in the present paper are different from technique used in the references cited earlier, and the results are the generalization of the existing results.

This paper is structured as follows: A few standard definitions and preliminaries are discussed in Section 2. Section 3 deals with new oscillation results for (1), and in Section 4 we provide suitable examples to demonstrate the main findings.

\section{Preliminaries}

In this section, some basic definitions and preliminary results are presented, which will be useful for further discussion.

We denote the polynomial factorial $\lambda_{\ell}^{(m)}$ by the expression

$$
\lambda_{\ell}^{(m)}=\lambda(\lambda-\ell)(\lambda-2 \ell) \cdots(\lambda-(m-1) \ell)=\ell^{m} \frac{\Gamma(1+\lambda / \ell)}{\Gamma(\lambda / \ell-(m-1))}
$$

Lemma 1 ([17]). Let $\ell \in[0, \infty)$. Then, $\Delta_{\ell}\left(\lambda_{\ell}^{(m)}\right)=(m \ell) \lambda_{\ell}^{(m-1)}$.

Lemma $2([17])$. Let $u(\lambda)$ and $v(\lambda)$ be any two real valued functions. Then,

$$
\Delta_{\ell}\{u(\lambda) v(\lambda)\}=u(\lambda+\ell) \Delta_{\ell} v(\lambda)+v(\lambda) \Delta_{\ell} u(\lambda)=v(\lambda+\ell) \Delta_{\ell} u(\lambda)+u(\lambda) \Delta_{\ell} v(\lambda) .
$$


Lemma 3. Let $y(\lambda)>0$ be a solution of (1). Then the function $x(\lambda)$ satisfies exactly one of the following two properties.

(i) $x(\lambda)>0, \Delta_{\ell} x(\lambda)>0, \Delta_{\ell}\left(p_{2}(\lambda) \Delta_{\ell} x(\lambda)\right)>0$;

(ii) $x(\lambda)>0, \Delta_{\ell} x(\lambda)<0, \Delta_{\ell}\left(p_{2}(\lambda) \Delta_{\ell} x(\lambda)\right)>0$,

where $\lambda \geq \lambda_{2}$ for large $\lambda_{2}$.

Proof. Let $\{y(\lambda)\}>0$ be a solution of Equation (1) for all $\lambda \geq \lambda_{0}$. Then from $x(\lambda)$, we have $x(\lambda) \geq y(\lambda)>0$ for $\lambda \geq \lambda_{1}$, and also from (1),

$$
\Delta_{\ell}\left(p_{1}(\lambda) \Delta_{\ell}\left(p_{2}(\lambda) \Delta_{\ell} x(\lambda)\right)\right)=-\sum_{s=c}^{d} h(\lambda, s) f(y(\lambda+s \ell-\sigma \ell))<0 .
$$

We know that $p_{1}(\lambda)\left(\Delta_{\ell}\left(p_{2}(\lambda)\left(\Delta_{\ell} x(\lambda)\right)\right)\right)$ is a decreasing function on $\left[\lambda_{1}, \infty\right)$ and it is either positive or negative eventually. It is possible to prove that $p_{1}(\lambda)\left(\Delta_{\ell}\left(p_{2}(\lambda)\left(\Delta_{\ell} x(\lambda)\right)\right)\right)>0$ for $\lambda \geq \lambda_{1} \geq \lambda_{0}$. If not, then there exists a constant $M_{1}>0$ such that

$$
\Delta_{\ell}\left(p_{2}(\lambda) \Delta_{\ell} x(\lambda)\right)<-\frac{M_{1}}{p_{1}(\lambda)}<0, \text { for } \lambda \geq \lambda_{1} .
$$

Hence, by (3)

$$
p_{2}(\lambda)\left(\Delta_{\ell} x(\lambda)\right) \leq p_{2}\left(\lambda_{1}\right)\left(\Delta_{\ell} x\left(\lambda_{1}\right)\right)-M_{1} \sum_{r=0}^{m_{1}(\lambda)} \frac{1}{p_{1}\left(\overline{\lambda_{1}}+r \ell\right)} .
$$

Letting $\lambda \rightarrow \infty$, and using condition $\left(c_{1}\right), \lim _{\lambda \rightarrow \infty} p_{2}(\lambda)\left(\Delta_{\ell} x(\lambda)\right)=-\infty$. Then we can find a $\lambda_{2} \geq \lambda_{1}$ and a constant $M_{2}>0$ with the condition

$$
p_{2}(\lambda)\left(\Delta_{\ell} x(\lambda)\right)<-M_{2}, \text { for } \lambda_{0} \geq \lambda
$$

Dividing the last inequality by $p_{2}(\lambda)$ and summing from $\lambda_{2}$ to $\lambda-\ell$, we get

$$
x(\lambda)<x\left(\lambda_{2}\right)-M_{2} \sum_{r_{1}=0}^{m_{2}(\lambda)} \frac{1}{p_{2}\left(\overline{\left.\lambda_{2}+r_{1} \ell\right)}\right.} .
$$

Letting $\lambda \rightarrow \infty$ and using condition $\left(c_{1}\right)$, we get $x(\lambda) \rightarrow-\infty$. That is, $x(\lambda)<0$ eventually, which is contradictory to $x(\lambda)>0$. Therefore, $\Delta_{\ell}\left(p_{2}(\lambda)\left(\Delta_{\ell} x(\lambda)\right)\right)$ is positive, that is $\Delta_{\ell}\left(p_{2}(\lambda)\left(\Delta_{\ell} x(\lambda)\right)\right)>$ 0 holds.

Since $\Delta_{\ell}\left(p_{2}(\lambda)\left(\Delta_{\ell} x(\lambda)\right)\right)>0, p_{2}(\lambda)\left(\Delta_{\ell} x(\lambda)\right)$ is monotonically increasing in the interval $\left[\lambda_{2}, \infty\right)$. Therefore, either $\Delta_{\ell} x(\lambda)>0$ or $\Delta_{\ell} x(\lambda)<0$ for all $\lambda$, which is either property (i) or (ii) for $\{x(\lambda)\}$.

Lemma 4. Let $\{y(\lambda)\}$ be a positive solution of Equation (1), and $x(\lambda)$ satisfies (ii) of Lemma 3. If

$$
\sum_{r_{2}=\lambda_{3}}^{\infty} \frac{1}{p_{2}\left(\overline{\lambda_{3}}+r_{2} \ell\right)}\left(\sum_{r_{1}=\lambda_{1}}^{m_{2}\left(r_{2}\right)} \frac{1}{p_{1}\left(\overline{\lambda_{2}}+r_{1} \ell\right)}\left(\sum_{r=0}^{m_{1}\left(r_{1}\right)} \sum_{s=c}^{d} h\left(\overline{\lambda_{1}}+r \ell, s\right)\right)\right)=\infty,
$$

then, the solution $y(\lambda)$ of Equation (1) tends to zero as $\lambda \rightarrow \infty$.

Proof. Let $\{y(\lambda)\}>0$ be a solution of Equation (1). From (ii) of Lemma 3, there exist $\gamma \geq 0$ with the condition

$$
0 \leq r=\lim _{\lambda \rightarrow \infty} x(\lambda)
$$


Now, we shall prove that $\gamma=0$. Let $\gamma>0, \gamma+\epsilon>x(\lambda)>\gamma \forall \epsilon>0$, where $\lambda$ is sufficiently large. Choosing $0<\epsilon<\frac{1-g}{g} \gamma$, from (2), it follows that

$$
\begin{aligned}
y(\lambda) & =x(\lambda)-\sum_{s=a}^{b} y(\lambda+s \ell-\tau \ell) g(\lambda, s) \\
& >\gamma-\sum_{s=a}^{b} y(\lambda+s \ell-\tau \ell) g(\lambda, s)>\gamma-g(\gamma+\epsilon)=\frac{\gamma-g(\gamma+\epsilon)}{\gamma+\epsilon}(\gamma+\epsilon)>M x(\lambda),
\end{aligned}
$$

where $M=\frac{\gamma-g(\gamma+\epsilon)}{\gamma+\epsilon}>0$. Hence, from Equation (1) and (c $\left.c_{3}\right)$, we have

$$
\Delta_{\ell}\left(p_{1}(\lambda) \Delta_{\ell}\left(p_{2}(\lambda) \Delta_{\ell} x(\lambda)\right)\right)=-\sum_{s=c}^{d} f(y(\lambda+s \ell-\sigma \ell)) h(\lambda, s) \leq-\sum_{s=c}^{d} L y(\lambda+s \ell-\sigma \ell) h(\lambda, s) .
$$

Now, using (8), we obtain

$$
\Delta_{\ell}\left(\Delta_{\ell}\left(p_{2}(\lambda) \Delta_{\ell} x(\lambda)\right) p_{1}(\lambda)\right) \leq-M L \sum_{s=c}^{d} h(\lambda, s) x(\lambda+s \ell-\sigma \ell) .
$$

Summing the last inequality form $\lambda_{1}$ to $\lambda-\ell$ and form (3), we get

$$
-p_{1}\left(\lambda_{1}\right) \Delta_{\ell}\left(p_{2}\left(\lambda_{1}\right) \Delta_{\ell} x\left(\lambda_{1}\right)\right) \leq-M L \sum_{r=0}^{m_{1}(\lambda)} \sum_{s=c}^{d} h\left(\overline{\lambda_{1}}+r \ell, s\right) x\left(\overline{\lambda_{1}}+r \ell+s \ell-\sigma \ell\right) .
$$

The above equation can also be written as

$$
\Delta_{\ell}\left(p_{2}\left(\lambda_{1}\right) \Delta_{\ell} x\left(\lambda_{1}\right)\right) \geq \frac{M L \gamma}{p_{1}\left(\lambda_{1}\right)} \sum_{r=0}^{m_{1}(\lambda)} \sum_{s=c}^{d} h\left(\overline{\lambda_{1}}+r \ell, s\right) .
$$

Summing again form $\lambda_{1}<\lambda_{2}$ to $\lambda-\ell$, we get

$$
-\Delta_{\ell} x\left(\lambda_{2}\right) \geq \frac{M L \gamma}{p_{2}\left(\lambda_{2}\right)} \sum_{r_{1}=\lambda_{1}}^{m_{2}(\lambda)} \frac{1}{p_{1}\left(\overline{\lambda_{2}}+r_{1} \ell\right)}\left(\sum_{r=0}^{m_{1}\left(r_{1}\right)} \sum_{s=c}^{d} h\left(\overline{\lambda_{1}}+r \ell, s\right)\right) .
$$

Summing the last inequality form $\lambda_{2}<\lambda_{3}$ to $\infty$, we get

$$
x\left(\lambda_{3}\right) \geq \operatorname{ML} \sum_{r_{2}=\lambda_{3}}^{\infty} \frac{1}{p_{2}\left(\overline{\lambda_{3}}+r_{2} \ell\right)}\left(\sum_{r_{1}=\lambda_{1}}^{m_{2}\left(r_{2}\right)} \frac{1}{p_{1}\left(\overline{\lambda_{2}}+r_{1} \ell\right)}\left(\sum_{r=0}^{m_{1}\left(r_{1}\right)} \sum_{s=c}^{d} h\left(\overline{\lambda_{1}}+r \ell, s\right)\right)\right) .
$$

This contradicts condition (7). Thus $\gamma=0$. Also, $0<y(\lambda)<x(\lambda)$ gives that $\lim _{\lambda \rightarrow \infty} x(\lambda)=0$. The proof is now complete.

Lemma 5. Let $y(\lambda)>0$ be a solution of Equation (1), and $x(\lambda)$ satisfies (i). Then

$$
\Delta_{\ell} x(\lambda) \geq \frac{p_{1}(\lambda+\ell) \Delta_{\ell}\left(p_{2}(\lambda+\ell) \Delta_{\ell} x(\lambda+\ell)\right) P(\lambda)}{p_{2}(\lambda)}
$$

where $P(\lambda)=\sum_{r=0}^{m_{1}(\lambda)} \frac{1}{p_{1}\left(\overline{\lambda_{1}+r \ell}\right)}$.

Proof. Let $y(\lambda)>0$ be a solution of Equation (1). Since $x(\lambda)$ satisfies (i),

$$
\Delta_{\ell}\left(p_{1}(\lambda) \Delta_{\ell}\left(p_{2}(\lambda) \Delta_{\ell} x(\lambda)\right)\right)<0 .
$$


From (3) and for all $\lambda_{1} \geq \lambda_{0}$, we have

$$
\begin{aligned}
\Delta_{\ell} x(\lambda) p_{2}(\lambda) & =\Delta_{\ell} x(\lambda) p_{2}(\lambda)+\sum_{r=0}^{m_{1}(\lambda)} \Delta_{\ell}\left(\Delta_{\ell} x\left(\overline{\lambda_{1}}+r \ell\right) p_{2}\left(\overline{\lambda_{1}}+r \ell\right)\right) \\
& \geq \sum_{r=0}^{m_{1}(\lambda)} \frac{p_{1}\left(\overline{\lambda_{1}}+r \ell\right) \Delta_{\ell}\left(p_{2}\left(\overline{\lambda_{1}}+r \ell\right) \Delta_{\ell} x\left(\overline{\lambda_{1}}+r \ell\right)\right)}{p_{1}\left(\overline{\lambda_{1}}+r \ell\right)} \\
& \geq \Delta_{\ell}\left(p_{2}(\lambda+\ell) \Delta_{\ell} x(\lambda+\ell)\right) p_{1}(\lambda+\ell) \sum_{r=0}^{m_{1}(\lambda)} \frac{1}{p_{1}\left(\overline{\lambda_{1}}+r \ell\right)} \\
& \geq \Delta_{\ell}\left(p_{2}(\lambda+\ell) \Delta_{\ell} x(\lambda+\ell)\right) p_{1}(\lambda+\ell) P(\lambda) .
\end{aligned}
$$

Hence, we obtain

$$
\Delta_{\ell} x(\lambda) \geq \frac{\Delta_{\ell}\left(p_{2}(\lambda+\ell) \Delta_{\ell} x(\lambda+\ell)\right) p_{1}(\lambda+\ell) P(\lambda)}{p_{2}(\lambda)},
$$

which completes the proof.

\section{Main Results}

In this section, we obtain new oscillation criteria for the Equation (1) by using the generalized Riccati transformation and Philos type technique. For Philos type technique, we define functions $q, Q: \mathbb{N}_{\ell} \times \mathbb{N}_{\ell} \rightarrow \mathbb{R}$ such that

(I) $Q(\lambda, \lambda)=0$ for $\lambda \geq \lambda_{1} \geq 0$;

(II) $Q(\lambda, s)>0$ for $\lambda>s \geq \lambda_{1}$;

(III) $\Delta_{\ell(s)} Q(\lambda, s)=Q(\lambda, s+\ell)-Q(\lambda, s) \leq 0$ for $\lambda>s \geq \lambda_{1}$ and a positive real sequence $\{\rho(\lambda)\}$ with the condition

$$
-q(\lambda, s) \sqrt{Q(\lambda, s)}=\Delta_{\ell(s)} Q(\lambda, s)+\frac{\Delta_{\ell} \rho(s)}{\rho(s+\ell)} Q(\lambda, s) .
$$

Theorem 1. Consider the Condition (7) and $\{\rho(\lambda)\}$ satisfies

$$
\sum_{r=0}^{m_{1}(\lambda)}\left(C\left(\overline{\lambda_{1}}+r \ell\right)-\frac{B^{2}\left(\overline{\lambda_{1}}+r \ell\right)}{4 A\left(\overline{\lambda_{1}}+r \ell\right)}\right)=\infty
$$

where

$$
A(\lambda)=\frac{\rho(\lambda) P(\lambda)}{\rho^{2}(\lambda+\ell) p_{2}(\lambda)}, \quad B(\lambda)=\frac{\Delta_{\ell} \rho(\lambda)}{\rho(\lambda+\ell)}, \quad C(\lambda)=H(\lambda) \rho(\lambda)
$$

and

$$
H(\lambda)=L(1-g) \sum_{s=c}^{d} h(\lambda, s),
$$

then, every solution of Equation (1) is either oscillatory or converges to zero.

Proof. Assume that $\{y(\lambda)\}$ is a non-oscillatory solution of Equation (1). Without loss of generality, we may assume that $y(\lambda)>0, y(\lambda+s \ell-\tau \ell)>0$ for $\lambda \geq \lambda_{1} \geq \lambda_{0} \in \mathbb{N}_{\ell}$ and $\{x(\lambda)\}$ is defined as in (2). Then $\{x(\lambda)\}$ will satisfy the two cases of Lemma 3.

Let $\{x(\lambda)\}$ satisfies property (i) of Lemma 3. From Equation (2), we have

$$
y(\lambda) \geq x(\lambda)-\sum_{s=a}^{b} x(\lambda+s \ell-\tau \ell) g(\lambda, s) \geq x(\lambda)\left(1-\sum_{s=a}^{b} g(\lambda, s)\right) \geq x(\lambda)(1-g) .
$$


Using condition $\left(c_{3}\right)$ in Equation (1),

$$
\left.\Delta_{\ell}\left(p_{1}(\lambda) \Delta_{\ell}\left(p_{2}(\lambda) \Delta_{\ell} x(\lambda)\right)\right) \leq-\sum_{s=c}^{d} h(\lambda, s) L x(\lambda+s \ell-\sigma \ell)\right) .
$$

Now, using Equation (15) in the above inequality, we obtain

$$
\begin{aligned}
\Delta_{\ell}\left(p_{1}(\lambda) \Delta_{\ell}\left(p_{2}(\lambda) \Delta_{\ell} x(\lambda)\right)\right) & \leq-L(1-p) \sum_{s=c}^{d} h(\lambda, s) x(\lambda+s \ell-\sigma \ell) \\
& \leq-H(\lambda) x(\lambda+c \ell-\sigma \ell) .
\end{aligned}
$$

The generalized Riccati transformation is

$$
w(\lambda)=\rho(\lambda) \frac{p_{1}(\lambda) \Delta_{\ell}\left(p_{2}(\lambda) \Delta_{\ell} x(\lambda)\right)}{x(\lambda)}, \quad \lambda \geq \lambda_{1} .
$$

Then, $w(\lambda)>0$ for all $\lambda \geq \lambda_{1}$, and Equations (10) and (17) yield

$$
\Delta_{\ell} w(\lambda) \leq-q_{1}(\lambda) \rho(\lambda)+w(\lambda+\ell) \frac{\Delta_{\ell} \rho(\lambda)}{\rho(\lambda+\ell)}-w^{2}(\lambda+\ell) \frac{\rho(\lambda) P(\lambda)}{\rho^{2}(\lambda+\ell) p_{2}(\lambda)} .
$$

The above equation is also expressed as

$$
\Delta_{\ell} w(\lambda) \leq-C(\lambda)+w(\lambda+\ell) B(\lambda)-w^{2}(\lambda+\ell) A(\lambda),
$$

where

$$
A(\lambda)=\frac{P(\lambda) \rho(\lambda)}{\rho^{2}(\lambda+\ell) p_{2}(\lambda)}, \quad B(\lambda)=\frac{\Delta_{\ell} \rho(\lambda)}{\rho(\lambda+\ell)}, \quad C(\lambda)=\rho(\lambda) H(\lambda) .
$$

Using the inequality

$$
A u-B u^{\frac{1+\beta}{\beta}} \leq \frac{\beta^{\beta}}{(1+\beta)^{1+\beta}} \times \frac{A^{1+\beta}}{B^{\beta}},
$$

Equation (20) can be written as

$$
C(\lambda)-\frac{B^{2}(\lambda)}{4 A(\lambda)} \leq-\Delta_{\ell} w(\lambda)
$$

Summing (22) from $\lambda_{1}$ to $\lambda-\ell$ gives

$$
\sum_{r=0}^{m_{1}(\lambda)}\left(C\left(\overline{\lambda_{1}}+r \ell\right)-\frac{B^{2}\left(\overline{\lambda_{1}}+r \ell\right)}{4 A\left(\overline{\lambda_{1}}+r \ell\right)}\right) \leq w\left(\lambda_{1}\right)-w(\lambda) \leq w\left(\lambda_{1}\right) .
$$

From $w(\lambda)>0$, we get a contradiction to (12), and hence $x(\lambda)$ is oscillatory. When $x(\lambda)$ satisfies property (ii), from (7) we obtain $\lim _{\lambda \rightarrow \infty} y(\lambda)=0$, which completes the proof.

Theorem 2. Consider the Condition (7) and let $\{\rho(\lambda)\}$ satisifies

$$
\limsup _{\lambda \rightarrow \infty} \frac{1}{Q(\lambda, s)} \sum_{r=0}^{\frac{\lambda-\bar{s}-\ell}{\ell}}\left[Q(\lambda, \bar{s}+r \ell) C(\bar{s}+r \ell)-\frac{h^{2}(\lambda, \bar{s}+r \ell)}{4 A(\bar{s}+r \ell)}\right]=\infty,
$$

then, every solution of Equation (1) is either oscillatory or converges to zero. 
Proof. Assume that $\{y(\lambda)\}$ is a non-oscillatory solution of Equation (1). As in Theorem 1, we have Equation (20). Now, if the Inequality (20) is multiplied by $Q(\lambda, s)$ and summing the resulting inequality form $\lambda_{2}$ to $\lambda-\ell$ for all $\lambda \geq \lambda_{2} \geq \lambda_{0}$, we obtain

$$
\begin{aligned}
& \sum_{r=0}^{m_{2}(\lambda)} Q\left(\lambda, \overline{\lambda_{2}}+r \ell\right) C\left(\overline{\lambda_{2}}+r \ell\right) \leq-\sum_{r=0}^{m_{2}(\lambda)} Q\left(\lambda, \overline{\lambda_{2}}+r \ell\right) \Delta_{\ell} w\left(\overline{\lambda_{2}}+r \ell\right) \\
& +\sum_{r=0}^{m_{2}(\lambda)}\left(B\left(\overline{\lambda_{2}}+r \ell\right) w\left(\overline{\lambda_{2}}+r \ell+\ell\right)-A\left(\overline{\lambda_{2}}+r \ell\right) w^{2}\left(\overline{\lambda_{2}}+r \ell+\ell\right)\right) Q\left(\lambda, \overline{\lambda_{2}}+r \ell\right) .
\end{aligned}
$$

By summation by parts,

$$
\begin{aligned}
\sum_{r=0}^{m_{2}(\lambda)} Q\left(\lambda, \overline{\lambda_{2}}+r \ell\right) C & \left.\overline{\lambda_{2}}+r \ell\right) \leq Q\left(\lambda, \lambda_{2}\right) w\left(\lambda_{2}\right) \\
& +\sum_{r=0}^{m_{2}(\lambda)}\left[Q\left(\bar{\lambda}, \overline{\lambda_{2}}+r \ell\right) \Delta_{\ell\left(\overline{\lambda_{2}}\right)}+Q\left(\lambda, \overline{\lambda_{2}}+r \ell\right) B\left(\overline{\lambda_{2}}+r \ell\right)\right] w\left(\overline{\lambda_{2}}+r \ell+\ell\right) \\
& -\sum_{r=0}^{m_{2}(\lambda)} Q\left(\lambda, \overline{\lambda_{2}}+r \ell\right) A\left(\overline{\lambda_{2}}+r \ell\right) w^{2}\left(\overline{\lambda_{2}}+r \ell+\ell\right) .
\end{aligned}
$$

Inequality (21) becomes

$$
\begin{aligned}
\sum_{r=0}^{m_{2}(\lambda)} Q\left(\lambda, \overline{\lambda_{2}}+r \ell\right) C\left(\overline{\lambda_{2}}+r \ell\right) \leq & Q\left(\lambda, \lambda_{2}\right) w\left(\lambda_{2}\right) \\
& +\sum_{r=0}^{m_{2}(\lambda)} \frac{\left[\Delta_{\ell\left(\overline{\lambda_{2}}\right)} Q\left(\lambda, \overline{\lambda_{2}}+r \ell\right)+B\left(\overline{\lambda_{2}}+r \ell\right) Q\left(\lambda, \overline{\lambda_{2}}+r \ell\right)\right]^{2}}{4 A\left(\overline{\lambda_{2}}+r \ell\right) Q\left(\lambda, \bar{\lambda}_{2}+r \ell\right)} .
\end{aligned}
$$

From (11), we have

$$
\begin{gathered}
\sum_{r=0}^{m_{2}(\lambda)}\left[C\left(\overline{\lambda_{2}}+r \ell\right) Q\left(\lambda, \overline{\lambda_{2}}+r \ell\right)-\frac{h^{2}\left(\lambda, \overline{\lambda_{2}}+r \ell\right)}{4 A\left(\overline{\lambda_{2}}+r \ell\right)}\right] \leq Q\left(\lambda, \lambda_{2}\right) w\left(\lambda_{2}\right) . \\
\frac{1}{Q\left(\lambda, \lambda_{2}\right)} \sum_{r=0}^{m_{2}(\lambda)}\left[C\left(\overline{\lambda_{2}}+r \ell\right) Q\left(\lambda, \overline{\lambda_{2}}+r \ell\right)-\frac{h^{2}\left(\lambda, \overline{\lambda_{2}}+r \ell\right)}{4 A\left(\overline{\lambda_{2}}+r \ell\right)}\right] \leq w\left(\lambda_{2}\right) .
\end{gathered}
$$

On letting $\lambda \rightarrow \infty$, (24) is contradictory. If $x(\lambda)$ satisfies property (ii) of Lemma 3 , then by condition (7) we have $\lim _{\lambda \rightarrow \infty} y(\lambda)=0$.

Corollary 1. If $(\lambda-s)_{\ell}^{(m)}=Q(\lambda, s)$ for all $0 \leq s \leq \lambda, \rho(\lambda)=1$ and

$$
\limsup _{\lambda \rightarrow \infty} \frac{1}{\lambda_{\ell}^{(m)}} \sum_{r=0}^{\frac{\lambda-\bar{s}-\ell}{\ell}}\left[(\lambda-\bar{s}-r \ell)_{\ell}^{(m)} C(\bar{s}+r \ell)-\frac{\left(m \ell(\lambda-\bar{s}-r \ell)_{\ell}^{(m-1)}\right)^{2}}{4 A(\bar{s}+r \ell) \sqrt{(\lambda-\bar{s}-r \ell)_{\ell}^{(m)}}}\right]=\infty,
$$

then for every $m \geq 1$, each solution of (1) is oscillatory or converges to 0 .

Corollary 2. If $Q(\lambda, s)=\left(\log \frac{\lambda+\ell}{s+\ell}\right)^{m} f \forall \lambda \geq s \geq 0, \rho(\lambda)=1$ and

$$
\limsup _{\lambda \rightarrow \infty} \frac{1}{(\log (\lambda+\ell))_{\ell}^{(m)}} \sum_{r=0}^{\frac{\lambda-\bar{s}-\ell}{\ell}}\left[\left(\log \frac{\lambda+\ell}{\bar{s}+r \ell+\ell}\right)^{m} C(\bar{s}+r \ell)-\frac{m\left(\log \frac{\lambda+\ell}{\bar{s}+r \ell+\ell}\right)^{\frac{m-2}{2}}}{4(\bar{s}+(r+1) \ell) A(\bar{s}+r \ell)}\right]=\infty,
$$

then, for every $m \geq 1$, each solution of (1) is oscillatory or converges to 0 .

Theorem 3. Assume that Condition (7) holds. Also, let

$$
0<\inf _{s \geq \lambda_{0}}\left[\liminf _{\lambda \rightarrow \infty} \frac{Q(\lambda, s)}{Q\left(\lambda, \lambda_{0}\right)}\right] \leq \infty
$$


and

$$
\limsup _{\lambda \rightarrow \infty} \frac{1}{Q\left(\lambda, \lambda_{0}\right)} \sum_{r=0}^{\frac{\lambda-\bar{s}-\ell}{\ell}} \frac{h^{2}(\lambda, \bar{s}+r \ell)}{A(\bar{s}+r \ell)}<\infty
$$

hold. If there is a sequence $\{\Phi(\lambda)\}$ such that

$$
\sum_{r=0}^{\frac{\lambda-\bar{s}-\ell}{\ell}} A(\bar{s}+r \ell) \Phi_{+}^{2}(\bar{s}+r \ell+\ell)=\infty
$$

and

$$
\limsup _{\lambda \rightarrow \infty} \frac{1}{Q(\lambda, s)} \sum_{r=0}^{\frac{\lambda-\overline{-}-\ell}{\ell}}\left[Q(\lambda, \bar{s}+r \ell) C(\bar{s}+r \ell)-\frac{h^{2}(\lambda, \bar{s}+r \ell)}{4 A(\bar{s}+r \ell)}\right] \geq \Phi(s),
$$

where

$$
\Phi_{+}(\bar{s}+r \ell+\ell)=\max \{\Phi(\bar{s}+r \ell+\ell), 0\} .
$$

Let $A(\lambda), B(\lambda), C(\lambda)$, and $Q(\lambda, s)$ be respectively defined in (13) and (11). Then, each solution of Equation (1) is either oscillatory or $y(\lambda) \rightarrow 0$, as $\lambda \rightarrow \infty$.

Proof. Let $y(\lambda)$ be a non oscillatory solution of Equation (1). As in Therorem 2, when $x(\lambda)$ satisfies property (i), from (26) and by rearranging the terms we obtain

$$
\begin{aligned}
w\left(\lambda_{2}\right) & \geq \limsup _{\lambda \rightarrow \infty} \frac{1}{Q\left(\lambda, \lambda_{2}\right)} \sum_{r=0}^{m_{2}(\lambda)}\left[C\left(\overline{\lambda_{2}}+r \ell\right) Q\left(\lambda, \overline{\lambda_{2}}+r \ell\right)-\frac{h^{2}\left(\lambda, \overline{\lambda_{2}}+r \ell\right)}{4 A\left(\overline{\lambda_{2}}+r \ell\right)}\right] \\
& +\liminf _{\lambda \rightarrow \infty} \frac{1}{Q\left(\lambda, \lambda_{2}\right)} \sum_{r=0}^{m_{2}(\lambda)}\left[\frac{Q\left(\lambda, \overline{\lambda_{2}}+r \ell\right)}{2 \sqrt{A\left(\overline{\lambda_{2}}+r \ell\right)}}+\sqrt{A\left(\overline{\lambda_{2}}+r \ell\right) Q\left(\lambda, \overline{\lambda_{2}}+r \ell\right)} w\left(\overline{\lambda_{2}}+r \ell+\ell\right)\right]^{2}
\end{aligned}
$$

for $\lambda \geq \lambda_{2}$. It follows from (35) that

$$
\begin{aligned}
& w\left(\lambda_{2}\right) \geq \Phi\left(\lambda_{2}\right) \\
& +\liminf _{\lambda \rightarrow \infty} \frac{1}{Q\left(\lambda, \lambda_{2}\right)} \sum_{r=0}^{m_{2}(\lambda)}\left[\frac{Q\left(\lambda, \overline{\lambda_{2}}+r \ell\right)}{2 \sqrt{A\left(\overline{\lambda_{2}}+r \ell\right)}}+\sqrt{A\left(\overline{\lambda_{2}}+r \ell\right) Q\left(\lambda, \overline{\lambda_{2}}+r \ell\right)} w\left(\overline{\lambda_{2}}+r \ell+\ell\right)\right]^{2}
\end{aligned}
$$

which implies,

$$
w\left(\lambda_{2}\right) \geq \Phi\left(\lambda_{2}\right)
$$

and then

$$
\liminf _{\lambda \rightarrow \infty} \frac{1}{Q\left(\lambda, \lambda_{2}\right)} \sum_{r=0}^{m_{2}(\lambda)}\left[\frac{Q\left(\lambda, \overline{\lambda_{2}}+r \ell\right)}{2 \sqrt{A\left(\overline{\lambda_{2}}+r \ell\right)}}+\sqrt{A\left(\overline{\lambda_{2}}+r \ell\right) Q\left(\lambda, \overline{\lambda_{2}}+r \ell\right)} w\left(\overline{\lambda_{2}}+r \ell+\ell\right)\right]^{2}<\infty .
$$

Therefore,

$$
\begin{aligned}
\liminf _{\lambda \rightarrow \infty} & {\left[\frac{1}{Q\left(\lambda, \lambda_{2}\right)} \sum_{r=0}^{m_{2}(\lambda)} Q\left(\lambda, \lambda_{2}+r \ell\right) A\left(\lambda_{2}+r \ell\right) w^{2}\left(\lambda_{2}+r \ell+\ell\right)\right.} \\
& +\sum_{r=0}^{m_{2}(\lambda)} q\left(\lambda, \lambda_{2}+r \ell\right) \sqrt{Q\left(\lambda, \lambda_{2}+r \ell\right)} w\left(\lambda_{2}+r \ell+\ell\right) \frac{1}{Q\left(\lambda, \lambda_{2}\right)} \\
& \left.+\frac{1}{4 Q\left(\lambda, \lambda_{2}\right)} \sum_{r=0}^{m_{2}(\lambda)} \frac{q^{2}\left(\lambda, \lambda_{2}+r \ell\right)}{\sqrt{A^{2}\left(\lambda_{2}+r \ell\right)}}\right]<\infty .
\end{aligned}
$$


Then,

$$
\begin{aligned}
\liminf _{\lambda \rightarrow \infty} & {\left[\sum_{r=0}^{m_{2}(\lambda)} Q\left(\lambda, \overline{\lambda_{2}}+r \ell\right) A\left(\overline{\lambda_{2}}+r \ell\right) w^{2}\left(\overline{\lambda_{2}}+r \ell+\ell\right) \frac{1}{Q\left(\lambda, \lambda_{2}\right)}\right.} \\
& \left.+\sum_{r=0}^{m_{2}(\lambda)} q\left(\lambda, \overline{\lambda_{2}}+r \ell\right) \sqrt{Q\left(\lambda, \overline{\lambda_{2}}+r \ell\right)} w\left(\overline{\lambda_{2}}+r \ell+\ell\right) \overline{Q\left(\lambda, \lambda_{2}\right)}\right]<\infty .
\end{aligned}
$$

The above inequality can be expressed as

$$
\liminf _{\lambda \rightarrow \infty}[U(\lambda)+V(\lambda)]<\infty \text { for } \lambda \geq \lambda_{2}
$$

where

$$
\begin{aligned}
U(\lambda) & =\sum_{r=0}^{m_{2}(\lambda)} Q\left(\lambda, \overline{\lambda_{2}}+r \ell\right) A\left(\overline{\lambda_{2}}+r \ell\right) w^{2}\left(\overline{\lambda_{2}}+r \ell+\ell\right) \frac{1}{Q\left(\lambda, \lambda_{2}\right)} \\
V(\lambda) & \left.=\sum_{r=0}^{m_{2}(\lambda)} q\left(\lambda, \overline{\lambda_{2}}+r \ell\right) \sqrt{Q\left(\lambda, \overline{\lambda_{2}}+r \ell\right.}\right) w\left(\overline{\lambda_{2}}+r \ell+\ell\right) \frac{1}{Q\left(\lambda, \lambda_{2}\right)} .
\end{aligned}
$$

We shall prove that

$$
\sum_{r=0}^{m_{2}(\lambda)} w^{2}\left(\overline{\lambda_{2}}+r \ell+\ell\right) A\left(\overline{\lambda_{2}}+r \ell\right)<\infty
$$

Suppose to the contrary that

$$
\sum_{r=0}^{m_{2}(\lambda)} A\left(\overline{\lambda_{2}}+r \ell\right) w^{2}\left(\lambda_{2}+r \ell+\ell\right)=\infty,
$$

from Equation (32), we have

$$
\inf _{s \geq \lambda_{0}}\left[\liminf _{\lambda \rightarrow \infty} \frac{Q(\lambda, s)}{Q\left(\lambda, \lambda_{0}\right)}\right]>\mu
$$

for $\mu>0$, then $\frac{Q(\lambda, s)}{Q\left(\lambda, \lambda_{0}\right)}>\mu$ for $\lambda \geq \lambda_{2} \geq \lambda_{1}$. Then, we can find a positive constant $M_{3}>0$ with the condition

$$
\sum_{r=0}^{m_{2}(\lambda)} w^{2}\left(\overline{\lambda_{2}}+r \ell+\ell\right) A\left(\overline{\lambda_{2}}+r \ell\right) \geq \frac{M_{3}}{\mu} .
$$

Thus for $\lambda \geq \lambda_{3}$ and using Equation (45), we obtain

$$
\begin{aligned}
U(\lambda)= & \frac{1}{Q\left(\lambda, \lambda_{2}\right)} \sum_{r=0}^{m_{2}(\lambda)} \Delta_{\ell}\left(\sum_{r_{1}=0}^{m_{2}(\lambda)} A\left(\overline{\lambda_{2}}+r_{1} \ell\right) w^{2}\left(\overline{\lambda_{2}}+r_{1} \ell+\ell\right)\right) Q\left(\lambda, \overline{\lambda_{2}}+r \ell\right) \\
& +A\left(\overline{\lambda_{2}}+r \ell\right) w^{2}\left(\overline{\lambda_{2}}+r \ell+\ell\right) \\
= & -\frac{1}{Q\left(\lambda, \lambda_{2}\right)} \sum_{r=0}^{m_{3}(\lambda)}\left(\sum_{r_{1}=0}^{m_{2}(r)} A\left(\overline{\lambda_{2}}+r_{1} \ell\right) w^{2}\left(\overline{\lambda_{2}}+r_{1} \ell+\ell\right)\right) \Delta_{\ell\left(\lambda_{3}\right)} Q\left(\lambda, \overline{\lambda_{3}}+r \ell\right) \\
& -\frac{A\left(\lambda_{2}\right) w^{2}\left(\lambda_{2}+\ell\right)}{Q\left(\lambda, \lambda_{2}\right)} \sum_{r=0}^{m_{3}(\lambda)} Q\left(\bar{\lambda}, \overline{\lambda_{3}}+r \ell\right)+A\left(\overline{\lambda_{2}}+r \ell\right) w^{2}\left(\overline{\lambda_{2}}+r \ell+\ell\right) \\
\geq & \frac{1}{Q\left(\lambda, \lambda_{2}\right)} \sum_{r=0}^{m_{3}(\lambda)}\left(\sum_{r_{1}=0}^{m_{2}(r)} A\left(\overline{\lambda_{2}}+r_{1} \ell\right) w^{2}\left(\overline{\lambda_{2}}+r_{1} \ell+\ell\right)\right)\left(-\Delta_{\ell}\left(\lambda_{3}\right) Q\left(\lambda, \overline{\lambda_{3}}+r \ell\right)\right) \\
\geq & \frac{M_{3}}{\mu Q\left(\lambda, \lambda_{2}\right)} \sum_{r=0}^{m_{3}(\lambda)}\left(-\Delta_{\ell\left(\lambda_{3}\right)} Q\left(\lambda, \overline{\lambda_{3}}+r \ell\right)\right) \geq \frac{M_{3} Q\left(\lambda, \lambda_{3}\right)}{\mu Q\left(\lambda, \lambda_{2}\right)} \geq M_{3} .
\end{aligned}
$$


Since $M_{3}$ is arbitrary,

$$
\lim _{\lambda \rightarrow \infty} U(\lambda)=\infty
$$

Next, consider a sequence $\left\{\lambda_{n}\right\}$ with the condition

$$
\lim _{n \rightarrow \infty}\left[U\left(\lambda_{n}\right)+V\left(\lambda_{n}\right)\right]=\liminf _{\lambda \rightarrow \infty}[Q(\lambda)+V(\lambda)] .
$$

It follows from (42) that we can find a number $M_{4}$ such that

$$
U\left(\lambda_{n}\right)+V\left(\lambda_{n}\right) \leq M_{4} \quad \forall n=0,1,2, \cdots .
$$

In view of (47), we conclude that

$$
\lim _{n \rightarrow \infty} V\left(\lambda_{n}\right)=-\infty
$$

By (49), for $n$ large enough, we have

$$
1+\frac{V\left(\lambda_{n}\right)}{U\left(\lambda_{n}\right)} \leq \frac{M_{2}}{U\left(\lambda_{n}\right)}<\frac{1}{2} .
$$

In view of (50), this implies that

$$
\lim _{n \rightarrow \infty} \frac{V^{2}\left(\lambda_{n}\right)}{U\left(\lambda_{n}\right)}=\infty
$$

On the other hand, by Schwarz's inequality, we have

$$
\begin{aligned}
V^{2}\left(\lambda_{n}\right)= & \left(\overline{Q\left(\lambda_{n}, \lambda_{2}\right)} \sum_{r=0}^{m_{2}\left(\lambda_{n}\right)} q\left(\lambda_{n}, \overline{\lambda_{2}}+r \ell\right) \sqrt{Q\left(\lambda_{n}, \overline{\lambda_{2}}+r \ell\right)} w\left(\overline{\lambda_{2}}+r \ell+\ell\right)\right)^{2} \\
\leq & \left(\frac{1}{Q\left(\lambda_{n}, \lambda_{2}\right)} \sum_{r=0}^{m_{2}\left(\lambda_{n}\right)} Q\left(\lambda_{n}, \overline{\lambda_{2}}+r \ell\right) A\left(\overline{\lambda_{2}}+r \ell\right) w^{2}\left(\overline{\lambda_{2}}+r \ell+\ell\right)\right) \\
& \left(\frac{1}{Q\left(\lambda_{n}, \lambda_{2}\right)} \sum_{r=0}^{m_{2}\left(\lambda_{n}\right)} \frac{q^{2}\left(\lambda_{n}, \overline{\lambda_{2}}+r \ell\right)}{A\left(\overline{\lambda_{2}}+r \ell\right)}\right) \leq U\left(\lambda_{n}\right)\left(\overline{\frac{1}{Q\left(\lambda_{n}, \lambda_{2}\right)}} \sum_{r=0}^{m_{2}\left(\lambda_{n}\right)} \frac{q^{2}\left(\lambda_{n}, \overline{\lambda_{2}}+r \ell\right)}{A\left(\overline{\lambda_{2}}+r \ell\right)}\right) .
\end{aligned}
$$

Consequently,

$$
\begin{aligned}
\frac{V^{2}\left(\lambda_{n}\right)}{U\left(\lambda_{n}\right)} & \leq \frac{1}{Q\left(\lambda_{n}, \lambda_{2}\right)} \sum_{r=0}^{m_{2}\left(\lambda_{n}\right)} \frac{q^{2}\left(\lambda_{n}, \overline{\lambda_{2}}+r \ell\right)}{A\left(\overline{\lambda_{2}}+r \ell\right)} \\
& \leq \frac{1}{\mu Q\left(\lambda_{n}, \lambda_{0}\right)} \sum_{r=0}^{m_{2}\left(\lambda_{n}\right)} \frac{q^{2}\left(\lambda_{n}, \overline{\lambda_{2}}+r \ell\right)}{A\left(\overline{\lambda_{2}}+r \ell\right)} .
\end{aligned}
$$

It follows from (52) that

$$
\lim _{n \rightarrow \infty} \frac{1}{Q\left(\lambda_{n}, \lambda_{0}\right)} \sum_{r=0}^{m_{2}\left(\lambda_{n}\right)} \frac{q^{2}\left(\lambda_{n}, \overline{\lambda_{2}}+r \ell\right)}{A\left(\overline{\lambda_{2}}+r \ell\right)}=\infty,
$$

which contradicts (33). Then, (43) holds. Hence, by (38)

$$
\sum_{r=0}^{m_{2}(\lambda)} A\left(\overline{\lambda_{2}}+r \ell\right) \Phi_{+}^{2}\left(\overline{\lambda_{2}}+r \ell+\ell\right) \leq \sum_{r=0}^{m_{2}(\lambda)} A\left(\overline{\lambda_{2}}+r \ell\right) w^{2}\left(\overline{\lambda_{2}}+r \ell+\ell\right)<\infty,
$$

which contradicts (34) and completes the proof. If $x(\lambda)$ satisfies property (ii) of Lemma 3, by Condition (7) we have $\lim _{\lambda \rightarrow \infty} y(\lambda)=0$. 
Theorem 4. Assume that all hypotheses of Theorem 3 are satisfied except condition (33). Also let

$$
\liminf _{\lambda \rightarrow \infty} \frac{1}{Q\left(\lambda, \lambda_{0}\right)} \sum_{r=0}^{\frac{\lambda-\bar{s}-\ell}{\ell}} Q(\lambda, \bar{s}+r \ell) C(\bar{s}+r \ell)<\infty
$$

and

$$
\liminf _{\lambda \rightarrow \infty} \frac{1}{Q(\lambda, s)} \sum_{r=0}^{\frac{\lambda-\bar{s}-\ell}{\ell}}\left[Q(\lambda, \bar{s}+r \ell) C(\bar{s}+r \ell)-\frac{q^{2}(\lambda, \bar{s}+r \ell)}{4 A(\bar{s}+r \ell)}\right] \geq \Phi(s),
$$

then, every solution of (1) is convergent to zero or oscillatory.

Proof. The proof is similar to that of Theorem 3 and hence the details are omitted.

Corollary 3. Let $m \geq 1$ be a constant, $\rho(\lambda)=1$. Suppose that

$$
\limsup _{\lambda \rightarrow \infty} \frac{1}{\lambda^{(m)}} \sum_{r=0}^{\frac{\lambda-\bar{s}-\ell}{\ell}} \frac{\left(m \ell(\lambda-\bar{s}-r \ell)_{\ell}^{(m-1)}\right)^{2}}{\sqrt{(\lambda-\bar{s}-r \ell)_{\ell}^{(m)}} A\left(\overline{\lambda_{2}}+r \ell\right)}<\infty .
$$

If there is a sequence $\{\Phi(\lambda)\}$ satisfying (34) and

$$
\limsup _{\lambda \rightarrow \infty} \frac{1}{(\lambda-s)_{\ell}^{(m)}} \sum_{r=0}^{\frac{\lambda-\bar{\ell}-\ell}{\ell}}\left[(\lambda-\bar{s}-r \ell)_{\ell}^{(m)} C(\bar{s}+r \ell)-\frac{\left(m \ell(\lambda-\bar{s}-r \ell)_{\ell}^{(m-1)}\right)^{2}}{4 A(\bar{s}+r \ell) \sqrt{(\lambda-\bar{s}-r \ell)_{\ell}^{(m)}}}\right] \geq \Phi(s),
$$

then, every solution of Equation (1) is oscillatory or converges to zero.

Corollary 4. Let $m \geq 1$ be a constant, and

$$
\liminf _{\lambda \rightarrow \infty} \frac{1}{\lambda^{(m)}} \sum_{r=0}^{m_{2}(\lambda)} m \ell\left(\lambda-\overline{\lambda_{2}}-r \ell\right)_{\ell}^{\left(\frac{m}{2}-1\right)} C\left(\overline{\lambda_{2}}+r \ell\right)<\infty .
$$

If there is a sequence $\{\Phi(\lambda)\}$ satisfying (34) and

$$
\liminf _{\lambda \rightarrow \infty} \frac{1}{(\lambda-s)_{\ell}^{(m)}} \sum_{r=0}^{\frac{\lambda-\bar{s}-\ell}{\ell}}\left[(\lambda-\bar{s}-r \ell)_{\ell}^{(m)} C(\bar{s}+r \ell)-\frac{\left(m \ell(\lambda-\bar{s}-r \ell)_{\ell}^{(m-1)}\right)^{2}}{4 A(\bar{s}+r \ell) \sqrt{(\lambda-\bar{s}-r \ell)_{\ell}^{(m)}}}\right] \geq \Phi(s),
$$

then every solution of Equation (1) will either oscillate or converge to 0.

\section{Examples}

Example 1. Consider the third order neutral generalized difference equation with distributed delay

$$
\Delta_{\ell}^{3}\left(y(\lambda)+\sum_{s=1}^{2} \frac{1}{4 s} y(\lambda+s \ell-\ell)\right)+\sum_{s=1}^{2} 9\left(\lambda+\frac{2}{s}\right) x(\lambda+s \ell-\ell)=0 .
$$

Here, $p_{1}(\lambda)=p_{2}(\lambda)=1, g(\lambda, s)=\frac{1}{4 s}, h(\lambda, s)=9\left(\lambda+\frac{2}{s}\right), \tau=\sigma=1$ and $\rho(\lambda)=1$. Then,$P(\lambda)=\frac{\lambda-\lambda_{1}}{\ell}$ and $H(\lambda)=\frac{45 L}{8}(2 \lambda+3)$, which implies

$$
\limsup _{\lambda \rightarrow \infty} \sum_{r=0}^{m_{1}(\lambda)} \frac{45 L}{8}\left(2\left(\overline{\lambda_{1}}+r \ell\right)+3\right)=\infty \quad \text { for } \quad 0 \leq L \leq 1
$$


It follows from Theorem 1 that every solution of Equation (61) is oscillatory. In fact, $\{y(\lambda)\}=\left\{(-1)^{\left[\frac{\lambda}{\ell}\right]}\right\}$ is one such oscillatory solution of Equation (61).

Example 2. Consider the third order neutral generalized difference equation with distributed delay

$$
\Delta_{\ell}^{2}\left(\lambda \Delta_{\ell}\left(y(\lambda)+\frac{1}{2} y(\lambda-2 \ell)+\frac{1}{4} y(\lambda-\ell)\right)\right)+y(\lambda) 10(\lambda+\ell)=0 .
$$

Here, $p_{1}(\lambda)=\lambda, p_{2}(\lambda)=1, g(\lambda, s)=\frac{1}{2 s}, h(\lambda, s)=10 s(\lambda+\ell), \tau=3, \sigma=1, a=c=d=1$ and $b=2$. Choose $\rho(\lambda)=1$ and $Q(\lambda, s)=(\lambda-s)_{\ell}^{(2)}$. By Theorem 2, $P(\lambda)=\frac{\lambda-\lambda_{1}}{\ell}, q(\lambda, s)=\frac{2 \ell \sqrt{\lambda-s}}{\sqrt{\lambda-s+\ell}}$, $H(\lambda)=\frac{5}{2}(\lambda+\ell), A(\lambda)=\frac{\lambda-\lambda_{1}}{\lambda \ell}, B(\lambda)=0$, and $C(\lambda)=\frac{5(\lambda+\ell)}{2}$ and

$$
\limsup _{\lambda \rightarrow \infty} \sum_{r=0}^{\frac{\lambda-s-\ell}{\ell}}\left[Q(\lambda, s+r \ell) C(s+r \ell)-\frac{h^{2}(\lambda, s+r \ell)}{4 A(s+r \ell)}\right] \frac{1}{Q(\lambda, s)}=\infty,
$$

Hence, by Theorem 2, every solution of Equation (62) is oscillatory. In fact, $\{y(\lambda)\}=\left\{(-1)^{\left[\frac{\lambda}{\ell}\right]}\right\}$ is one such oscillatory solution of Equation (62).

\section{Conclusions}

In this paper, we present new oscillation criteria for the generalized difference equation with distributed delay, which is new in the literature. Similar results are available in the literature for difference equations with delay involving the conventional difference operator $\Delta$. The results we obtained in this paper for difference equations involving the generalized difference operator $\Delta_{\ell}$ with distributed delay are rare and new in the literature. Also, the techniques we adopted are different from the techniques adopted by other researchers. Our results generalize the results on the oscillatory behavior of the continuous third order dynamical systems discussed in [5]. In addition, our results generalize the results presented in [18]. Other researchers considered only the oscillation criteria, but we obtained conditions for the solutions to be convergent apart form obtaining oscillation results.

Author Contributions: The authors contributed equally and all authors read the manuscript and approved the final submission.

Funding: This research received no external funding.

Acknowledgments: The authors would like to thank the referees for the valuable comments that helped to improve the manuscript.

Conflicts of Interest: The authors declare no conflict of interest.

\section{References}

1. Agarwal, R.P. Difference Equations and Inequalities, Theory, Methods and Applications, 2nd ed.; Marcel Dekker: New York, NY, USA, 2000.

2. Levi, D.; Termblay, S.; Winterniz, P. Lie point symmetries of difference equations and lattices. J. Phys. A Math. Gen. 2001, 33, 8501-8523. [CrossRef]

3. Levi, D.; Termblay, S.; Winterniz, P. Lie symmetries of multidimensional difference equations. J. Phys. A Math. Gen. 2001, 34, 9507-9524. [CrossRef]

4. Levi, D.; Winterniz, P. Continuous symmetries of difference equations. J. Phys. A: Math. Gen. 2005, 39, R1-R63. [CrossRef]

5. Grace, S.R.; Graef, J.R.; Tunc, E. Oscillatory behavior of third order nonlinear differential equations with nonlinear nonpositive neutral term. J. Taibah Univ. Sci. 2019, 13, 704-710. [CrossRef]

6. Agarwal, R.P.; Bohner, M.; Grace, S.R.; O’Regan. D. Discrete Oscillation Theory; Hindawi Publishers: New York, NY, USA, 2005. 
7. Gyori, I.; Ladas, G. Oscillation Theory of Delay Differential Equations with Applications; Clarendon Press: Oxford, UK, 1991.

8. Elaydi, S.N. An Introduction to Difference Equations, 3rd ed.; Springer: New York, NY, USA, 2000.

9. Schmeidal, E. Oscillatory and asymptotically zero soluions of third order difference equations with quasi differences. Opuscula Math. 2006, 26, 361-369.

10. Aktas, M.F.; Tiryaki, A.; Zafer, A. Oscillation of the third-order nonlinear delay difference equations. Turk. J. Math. 2012, 36, 422-436.

11. Elabbasy, E.M.; Barsom, M.Y.; AL-dheleai, F.S. New Oscillation Criteria for Third-Order Nonlinear Mixed Neutral Difference Equations. Chin. J. Math. 2014, 2014, 676470. [CrossRef]

12. Saker, S.H.; Alzabut, J.O.; Mukheimer, A. On the oscillatory behavior for a certain class of third order nonlinear delay difference equations. Electron. J. Qual. Theory Differ. Equ. 2010, 67, 1-16. [CrossRef]

13. Selvaraj, B.; Jaffer, I.M.A. On the oscillation of the solution to third order nonlinear difference equations. J. Math. Comput. Sci. 2010, 7, 873-876.

14. Selvaraj, B.; Mohankumar, P.; Balasubramanian, A. Oscillatory solutions of certain third order non-linear difference equations. Int. J. Nonlinear Sci. 2012, 14, 216-219.

15. Selvaraj, B.; Mohankumar, P.; Ananthan, V. Oscillatory and non oscillatory behavior of neutral delay difference equations. Int. J. Nonlinear Sci. 2012, 13, 472-474.

16. Thandapani, E.; Selvarangam, S.; Seghar, D. Oscillatory behavior of third order nonlinear difference equation with mixed neutral terms. Electron. J. Qual. Theory Differ. Equ. 2014, 53, 1-11. [CrossRef]

17. Maria Susai Manuel, M.; Britto Antony Xavier, G.; Thandapani, E. Theory of generalized difference operator and its applications. Far East J. Math. Sci. 2006, 20, 163-171.

18. Dinakar, P.; Selvarangan, S.; Thandapani, E. Oscillation of non linear third order perturbed functional difference equations. Nonautonomous Dyn. Syst. 2019, 6, 57-64. [CrossRef]

(C) 2019 by the authors. Licensee MDPI, Basel, Switzerland. This article is an open access article distributed under the terms and conditions of the Creative Commons Attribution (CC BY) license (http:/ / creativecommons.org/licenses/by/4.0/). 